\title{
Corrigendum to "Summarizing Online Movie Reviews: A Machine Learning Approach to Big Data Analytics"
}

\author{
Atif Khan $\left(\mathbb{D},{ }^{1}\right.$ Muhammad Adnan Gul ${ }^{D},{ }^{1}$ M. Irfan Uddin ${ }^{D},{ }^{2}$ Syed Atif Ali Shah $\left(\mathbb{D},{ }^{3}\right.$ \\ Shafiq Ahmad $\mathbb{D}^{4}{ }^{4}$ Muhammad Dzulqarnain Al Firdausi, ${ }^{4}$ and Mazen Zaindin ${ }^{5}$ \\ ${ }^{1}$ Department of Computer Science, Islamia College Peshawar, Peshawar, Pakistan \\ ${ }^{2}$ Institute of Computing, Kohat University of Science and Technology, Kohat, Pakistan \\ ${ }^{3}$ Faculty of Engineering and Information Technology, Northern University, Nowshehra, Pakistan \\ ${ }^{4}$ Industrial Engineering Department, College of Engineering, King Saud University, P.O. Box 800, Riyadh 11421, Saudi Arabia \\ ${ }^{5}$ Department of Statistics and Operations Research, College of Science, King Saud University, P.O. Box 2455, Riyadh 11451, \\ Saudi Arabia
}

Correspondence should be addressed to Shafiq Ahmad; ashafiq@ksu.edu.sa

Received 18 December 2020; Accepted 18 December 2020; Published 9 January 2021

Copyright (c) 2021 Atif Khan et al. This is an open access article distributed under the Creative Commons Attribution License, which permits unrestricted use, distribution, and reproduction in any medium, provided the original work is properly cited.

In the article titled "Summarizing Online Movie Reviews: A Machine Learning Approach to Big Data Analytics" [1], a citation was missing to the authors' recent related work [2]. At the request of the authors, the article has been corrected inline to reduce the similarity and include a discussion of how the methods compare.

\section{References}

[1] A. Khan, M. A. Gul, M. Irfan Uddin et al., "Summarizing online movie reviews: a machine learning approach to big data analytics," Scientific Programming, vol. 2020, Article ID 5812715, 14 pages, 2020.

[2] A. Khan, M. A. Gul, M. Zareei et al., "Movie review summarization using supervised learning and graph-based ranking algorithm," Computational Intelligence and Neuroscience, vol. 2020, Article ID 7526580, 14 pages, 2020. 